\title{
CONVERGENCE OF SERIES OF DILATED FUNCTIONS AND SPECTRAL NORMS OF GCD MATRICES
}

\author{
CHRISTOPH AISTLEITNER, ISTVÁN BERKES, KRISTIAN SEIP, AND MICHEL WEBER
}

\begin{abstract}
In the present paper we establish a connection between the $L^{2}$ norm of sums of dilated functions whose Fourier coefficients are of order $\mathscr{O}\left(j^{-\alpha}\right)$ for some $\alpha \in(1 / 2,1)$, and the spectral norms of certain greatest common divisor (GCD) matrices. Utilizing recent bounds for these spectral norms, we obtain necessary and sufficient conditions for the convergence in $L^{2}$ and for the almost everywhere convergence of series of dilated functions.
\end{abstract}

\section{INTRODUCTION}

Let $\left(c_{k}\right)_{k \geq 1}$ be a sequence of real numbers. Then Carleson's theorem [11] states that the series

$$
\sum_{k=1}^{\infty} c_{k} \sin 2 \pi k x \quad \text { and } \quad \sum_{k=1}^{\infty} c_{k} \cos 2 \pi k x
$$

are convergent for almost every $x$ in $[0,1]$ provided that $\left(c_{k}\right)_{k \geq 1}$ satisfies

$$
\sum_{k=1}^{\infty} c_{k}^{2}<\infty
$$

By orthogonality, condition (2) is also necessary and sufficient for the $L^{2}$ norm convergence of the two series in (1). A much studied problem is what happens in the previous convergence problems if the functions $\sin 2 \pi x$ and $\cos 2 \pi x$ are replaced by more general periodic functions. More precisely, the question is what we can say about the convergence of the series

$$
\sum_{k=1}^{\infty} c_{k} f(k x)
$$

when $f: \mathbb{R} \rightarrow \mathbb{R}$ is a measurable function satisfying

$$
f(x+1)=f(x), \quad \int_{0}^{1} f(x) d x=0, \quad \int_{0}^{1} f^{2}(x) d x<\infty .
$$

In general, (2) will not be a sufficient condition either for convergence in $L^{2}$ or for almost everywhere convergence of (3), and the problem is to find alternate conditions on the coefficients $\left(c_{k}\right)_{k \geq 1}$ when $f$ belongs to a prescribed class of functions. For a survey of existing

2010 Mathematics Subject Classification. 42A16, 42A20, 42A61, 42B05, 11A05, 15A18, 26 A45.

The first author is supported by a Schrödinger scholarship of the Austrian Research Foundation (FWF). The second author is supported by FWF Grant P 24302-N18 and OTKA grant K 108615. The third author is supported by the Research Council of Norway grant 227768. This paper was initiated while three of the authors (Berkes, Seip, Weber) participated in the research program Operator Related Function Theory and Time-Frequency Analysis at the Centre for Advanced Study at the Norwegian Academy of Science and Letters in Oslo during 2012-2013. 
results in the literature in this direction and recent results we refer to [2], [6].

In this paper, we will be interested in the case when $f$ belongs to the class $C_{\alpha}$ for $\alpha>1 / 2$, i.e. when the Fourier series of $f$ is of the form

$$
\sum_{j=1}^{\infty}\left(a_{j} \sin 2 \pi j x+b_{j} \cos 2 \pi j x\right)
$$

with

$$
\left|a_{j}\right|=\mathscr{O}\left(j^{-\alpha}\right), \quad\left|b_{j}\right|=\mathscr{O}\left(j^{-\alpha}\right), \quad \text { as } j \rightarrow \infty .
$$

The important limiting case $\alpha=1$ is essentially covered by the results of [2] (see Section 3 for details). We will now extend the methods of [2] to cover also the range $1 / 2<\alpha<1$ and will give necessary and sufficient conditions for the $L^{2}$ convergence and the almost everywhere convergence of (3) as well as of the related series

$$
\sum_{k=1}^{\infty} c_{k} f\left(n_{k} x\right)
$$

where $\left(n_{k}\right)_{k \geq 1}$ is a sequence of distinct positive integers.

Problems concerning the convergence of (3) or (5) can be traced back to Riemann's Habilitationsschrift (1852). They exhibit profound interrelations between various parts of analysis and number theory, as illustrated by the following list of important contributions: classical formulas of Franel and Landau connecting the convergence theory of (3) and (5) to sums of greatest common divisors (GCD sums); their generalization to the Hurwitz zeta function due to Mikolás; the work of Koksma, Erdős, Gál, LeVeque, and others in Diophantine approximation and uniform distribution theory; the results of Dyer and Harman in the context of the DuffinSchaeffer conjecture in metric Diophantine approximation; upper and lower bounds for GCD sums obtained by the authors of the present paper; and problems concerning the magnitude of the largest eigenvalue of GCD matrices, which were studied by Wintner, by Lindqvist and Seip (in the context of questions about Riesz bases), and by Hilberdink (in the context of the Riemann zeta function). Basic work on the convergence and divergence of dilated series and their relation to lacunary series was done by Gaposhkin, Nikishin, Philipp, and Kaufman, just to mention a few. An extensive survey on convergence problems for sums of dilated functions can be found in [6].

In view of this multitude of connections, we have found it appropriate to give a fairly detailed presentation of those ideas and lines of research that are most relevant for our particular problem. To this end, following the statement of our three main theorems in the next section, Section 3 gives an extensive survey of relevant background material. Section 4 contains auxiliary results, and proofs are given in Section 5. 


\section{RESUlts}

Throughout this paper we write $K, \hat{K}, K_{1}, K_{2}, \ldots$ for appropriate positive constants, not always the same, which only depend (at most) on $\alpha$ and $f$. We will use the Vinogradov symbols "«" and " $\gg$ " in the same sense. Throughout this paper, we assume that $\left(c_{k}\right)_{1 \leq k \leq N}$ and $\left(c_{k}\right)_{k \geq 1}$ denote sequences of real numbers and that $\left(n_{k}\right)_{1 \leq k \leq N}$ and $\left(n_{k}\right)_{k \geq 1}$ denote sequences of distinct positive integers. For notational convenience, throughout this paper we will read $\log x$ as $\max \{1, \log x\}$; in particular this implies that iterated logarithms are defined and non-zero.

Theorem 1. Assume that $f \in C_{\alpha}$ for some $\alpha \in(1 / 2,1)$. Then the series (3) is convergent in $L^{2}$ norm as well as almost everywhere convergent provided

$$
\sum_{k=1}^{\infty} c_{k}^{2} \exp \left(\frac{K(\log k)^{1-\alpha}}{\log \log k}\right)<\infty, \quad \text { where } K=3 /(1-\alpha)+4 / \sqrt{2 \alpha-1} .
$$

Conversely, for every $\alpha \in(1 / 2,1)$ there exist a function $f \in C_{\alpha}$ and a sequence $\left(c_{k}\right)_{k \geq 1}$ such that (6) holds with $K$ replaced by $(1-\varepsilon) /(1-\alpha)$ for every $0<\varepsilon<1$, but the series (3) is not convergent in $L^{2}$.

Theorem 2. Assume that $f \in C_{\alpha}$ for some $\alpha \in(1 / 2,1)$. Then the series (5) is convergent in $L^{2}$ norm as well as almost everywhere convergent if

$$
\sum_{k=1}^{\infty} c_{k}^{2} \exp \left(\frac{K(\log k)^{1-\alpha}}{(\log \log k)^{\alpha}}\right)<\infty, \quad \text { where } K=6 /(1-\alpha)+7\left(|\log (2 \alpha-1)|^{1 / 2}+1\right) .
$$

Conversely, for every $\alpha \in(1 / 2,1)$ there exist a function $f \in C_{\alpha}$, a sequence $\left(c_{k}\right)_{k \geq 1}$, a sequence $\left(n_{k}\right)_{k \geq 1}$, and a constant $\hat{K}=\hat{K}(\alpha)$ such that (7) holds with $K$ replaced by $\hat{K}$, but the series (5) is not convergent in $L^{2}$ and is divergent almost everywhere.

Theorem 1 improves results of Brémont [10], who proved that (3) is convergent in $L^{2}$ norm and almost everywhere provided

$$
\sum_{k=1}^{\infty} c_{k}^{2} \exp \left(\frac{(1+\varepsilon)(\log k)^{2-2 \alpha}}{2(1-\alpha) \log \log k}\right)<\infty \quad \text { for some } \varepsilon>0
$$

Brémont also proved that there exists a sequence $\left(c_{k}\right)_{k \geq 1}$ satisfying (2) such that the series (3) does not converge in $L^{2}$ and is almost everywhere divergent.

As the second part of Theorem 2 shows, condition (7) is essentially optimal both for convergence in $L^{2}$ and almost everywhere convergence, except for the precise value of the constant, thus providing a nearly complete solution of the problem of norm convergence and almost everywhere convergence of series of the form (5). In Theorem 1, we claim the necessity of condition (6) only for the norm convergence of (3); we do not know whether (6) is necessary also for almost everywhere convergence. However, we know that, in general, condition (2) is not sufficient for the almost everywhere convergence of the series (3). This follows from our proof of the optimality of the convergence condition in Theorem 2 for almost everywhere convergence of (5). In fact, for the proof of the optimality of Theorem 2 for given $\alpha \in(1 / 2,1)$ and an appropriate function $f \in C_{\alpha}$ we construct sequences $\left(c_{k}\right)_{k \geq 1}$ and $\left(n_{k}\right)_{k \geq 1}$ such that 
condition (7) holds for a certain value of $K$, but the series (5) is almost everywhere divergent. The proof reveals that $n_{k}$ is of asymptotic order at most $R^{k \log k}$ for some constant $R=R(\alpha)$. Consequently, setting $d_{n_{k}}=c_{k}$ when $n=n_{k}$ and $d_{n}=0$ otherwise, we see that $\sum_{n=1}^{\infty} d_{n} f(n x)$ is divergent almost everywhere, but

$$
\begin{aligned}
\sum_{n=1}^{\infty} d_{n} \exp \left(\frac{\hat{K}(\log \log n)^{1-\alpha}}{(\log \log \log n)^{\alpha}}\right) & =\sum_{k=1}^{\infty} d_{n_{k}} \exp \left(\frac{\hat{K}\left(\log \log n_{k}\right)^{1-\alpha}}{\left(\log \log \log n_{k}\right)^{\alpha}}\right) \\
& \leq \sum_{k=1}^{\infty} c_{k} \exp \left(\frac{K(\log k)^{1-\alpha}}{(\log \log k)^{\alpha}}\right)<\infty
\end{aligned}
$$

for some (sufficiently small) positive constant $\hat{K}$. Hence, in the condition for almost everywhere convergence in Theorem 1, a Weyl factor of order at least

$$
\exp \left(\frac{\hat{K}(\log \log k)^{1-\alpha}}{(\log \log \log k)^{\alpha}}\right)
$$

is necessary. This leaves a rather large gap in comparison to the Weyl factor in (6).

As noted, Theorem 1 gives a nearly optimal condition for the problem of $L^{2}$ convergence of series of the form (3). More precisely, this statement is true as long as one requests the extra convergence factor to be a "simple", slowly varying function. On the other hand, the situation is totally different if one allows the extra convergence function $\psi(k)$ to depend on numbertheoretic properties of $k$ and to be strongly fluctuating as $k$ increases. In this sense, Theorem 1 may be said to conceal the arithmetical nature of our problem. To state the next result, we introduce the divisor function

$$
\sigma_{s}(k)=\sum_{d \mid k} d^{s}
$$

We will prove the following result.

Theorem 3. Assume that $f \in C_{\alpha}$ for some $\alpha \in(1 / 2,1)$. Assume also that

$$
\sum_{k=1}^{\infty} c_{k}^{2} \sigma_{1-2 \alpha+\varepsilon}(k)<\infty
$$

for some $\varepsilon>0$. Then (3) is convergent in $L^{2}$. On the other hand, for every $\alpha \in(1 / 2,1)$ and every $0<\beta<1$ there exist a function $f \in C_{\alpha}$ and a real sequence $\left(c_{k}\right)_{k \geq 1}$ such that

$$
\sum_{k=1}^{\infty} c_{k}^{2} \sigma_{-\alpha}(k)^{\beta}<\infty,
$$

but (3) is not convergent in $L^{2}$.

In Berkes and Weber [5] it is proved that

$$
\sum_{k=1}^{\infty} c_{k}^{2} \sigma_{1-2 \alpha}(k)(\log k)^{2}<\infty
$$


implies the $L^{2}$ convergence and almost everywhere convergence of (3). Despite the similarity of (8) and (10), there is a crucial difference between the corresponding convergence statements. Clearly, for every $s>0$ we have

$$
\sum_{k=1}^{n} \sigma_{-s}(k)=\sum_{k=1}^{n} \sum_{d \mid k} d^{-s}=\sum_{d=1}^{\infty}\left\lfloor\frac{n}{d}\right\rfloor d^{-s} \sim n \sum_{d=1}^{\infty} d^{-1-s} \quad \text { as } n \rightarrow \infty,
$$

showing that the average value of the function $\sigma_{-s}(k)$ is $\sum_{d=1}^{\infty} d^{-1-s}<\infty$. This implies that given any function $\omega(k) \rightarrow \infty$, the asymptotic density of the set $\left\{k: \sigma_{-s}(k) \leq \omega(k)\right\}$ is 1 and thus for $\alpha>1 / 2$ and sufficiently small $\varepsilon>0$, the Weyl factor $\sigma_{1-2 \alpha+\varepsilon}(k)$ in (8) is of order $\mathscr{O}(\omega(k))$ for "most" $k$. Thus, despite the necessity of the condition

$$
\sum_{k=1}^{\infty} c_{k}^{2} \exp \left(\frac{K(\log k)^{1-\alpha}}{\log \log k}\right)<\infty
$$

in Theorem 1, for most $k$ the much smaller Weyl factor $\omega(k)$ suffices for the norm convergence of $\sum_{k=1}^{\infty} c_{k} f(k x)$. This effect will be apparent from the proofs of the divergence results in Theorems 1-3. The construction of $\left(c_{k}\right)_{k \geq 1}$ and $\left(n_{k}\right)_{k \geq 1}$ in the divergent examples uses, roughly speaking, the eigenvectors of suitable GCD matrices belonging to the maximal eigenvalue, which, as is seen from [2] and [16], are concentrated on indices $k$ with many small prime factors. These are also the indices $k$ where the divisor functions $\sigma_{-s}(k)$ are large: as Gronwall [15] showed,

$$
\sigma_{-s}(k) \leq \exp \left(\frac{1+o(1)}{1-s} \frac{(\log k)^{1-s}}{\log \log k}\right)
$$

and $\sigma_{-s}(k)$ reaches the order of magnitude on the right hand side along the sequence $k_{r}=$ $p_{1} \cdots p_{r}, r=1,2, \ldots$, where $\left(p_{r}\right)_{r \geq 1}$ is the sequence of primes. There is a gap between (8) and (9) and the optimal arithmetic function required for the $L^{2}$ norm convergence of (3) remains open.

As mentioned in the introduction, the case $\alpha=1$ is essentially covered by the results of [2]. We refer here to [2, Theorem 4], concerning the almost everywhere convergence of (5) for functions $f$ of bounded variation. The only property used in the proof of that result is that a function of bounded variation belongs to $C_{1}$. It therefore follows from [2, Theorem 4] that (5) is almost everywhere convergent when $f \in C_{1}$, provided that

$$
\sum_{k=1}^{\infty} c_{k}^{2}(\log \log k)^{\gamma}<\infty
$$

for some $\gamma>4$ (under the additional assumption that $\left(n_{k}\right)_{k \geq 1}$ is strictly increasing). Moreover, it was proved in [2] that this statement becomes false for $\gamma<2$. Using the results from [13] and the method of the proof of the second part of Theorem 2 of the present paper, it is possible to obtain the somewhat easier result that the series (3) and (5) are convergent in $L^{2}$ for all $f \in C_{1}$ if and only if

$$
\sum_{k=1}^{N} c_{k}^{2}(\log \log k)^{2}<\infty
$$


The problem of norm and a.e. convergence of (3) when for $f$ we assume only (4) is considerably harder. The reason of the difficulties is that while for $f \in C_{\alpha}$ we have

$$
\left|\int_{0}^{1} f(k x) f(\ell x) d x\right| \leq C \frac{(\operatorname{gcd}(k, \ell))^{2 \alpha}}{(k \ell)^{\alpha}}, \quad k, \ell \geq 1
$$

for some constant $C>0$, for general $f$ satisfying (4) the integral in (13) depends on $k, \ell$ and the Fourier coefficients of $f$ in a rather complicated way and the arithmetic machinery involving GCD sums and eigenvalues of GCD matrices used in the proof of our theorems breaks down. Assuming that the complex Fourier coefficients $a_{n}$ of $f$ satisfy $\left|a_{n}\right| \leq \phi(n)$, where the positive function $\phi$ has the homogeneity property $|\phi(n k)| \ll k^{-\gamma} \phi(n)$ for some $\gamma>0$, much of what is developed in the present paper will carry over to this situation. Estimates as those found in [8] could then, for instance, be used to obtain to get fairly sharp analogues of Theorem 1 and Theorem 2 for the considered function classes. We will return to this question in a subsequent paper.

In case of arithmetic criteria like in Theorem 3, Berkes and Weber [7] proved that if $f$ satisfies (4) with complex Fourier coefficients $a_{k}$, then the series (3) converges a.e. provided

$$
\sum_{k=1}^{\infty} c_{k}^{2} \psi(k)(\log k)^{2}<\infty
$$

where the arithmetic function $\psi$ is defined by

$$
\psi(n)=\sum_{d \mid n}(d g(d)+G(d)) \quad \text { where } \quad g(r)=\sum_{k=1}^{\infty}\left|a_{r k}\right|^{2}, \quad G(r)=\sum_{j \leq 2} g(j) .
$$

For example, if $\left|a_{k}\right| \leq C k^{-1 / 2}(\log k)^{-\gamma}, \gamma>1 / 2$, then $\psi(k)$ reduces to

$$
\psi(k)=\sum_{d \mid k}(\log d)^{-(2 \gamma-1)} .
$$

Note that the arithmetic function $\psi$ in (16) is larger than the one in (8), which is of course to be expected. Note also that if $k^{-\gamma}\left|a_{k}\right|$ is non-increasing for some $\gamma>0$, then in (14) we can choose

$$
\psi(k)=d(k)=\sum_{d \mid k} 1 .
$$

The same criterion holds if $f$ belongs to the Lip $(\alpha)$ class for some $\alpha>0$, see [5], [30]. These remarks show again the strong arithmetic character of our convergence problem. In [7] it is also shown that except the factor $(\log k)^{2}$, condition (14) is optimal. However, just like in Theorem 3, the arithmetic criterion (14) is not as sharp as the ones in Theorems 1 and 2.

Note that if (3) converges a.e. for $c_{k}=1 / k$, then by the Kronecker lemma we have

$$
\lim _{N \rightarrow \infty} \frac{1}{N} \sum_{k=1}^{N} f(k x)=0 \quad \text { a.e. }
$$

and thus the a.e. convergence problem of (3) under (4) is closely connected with the classical problem of the convergence of averages in (17). Khinchin [19] conjectured that under (4) 
(even without the third condition) the convergence relation (17) holds. This conjecture was disproved nearly 50 years later by a famous counterexample of Marstrand [25]. In the positive direction, Koksma [22] proved that (17) holds provided the complex Fourier coefficients $a_{k}$ of $f$ satisfy

$$
\sum_{k=1}^{\infty}\left|a_{k}\right|^{2} \sigma_{-1}(k)<\infty
$$

Bourgain [9] gave a new, much simplified counterexample in Khinchin's conjecture and claimed, without proof, that Koksma's criterion is essentially optimal. This claim was proved recently by Berkes and Weber [7]. Thus while the a.e. convergence problem for (3) under (4) remains open, the closely related problem of a.e. convergence of averages (17) is essentially settled.

\section{THE ROLE OF GCD MATRICES AND CERTAIN EXTREMAL FUNCTIONS IN $C_{\alpha}$}

We will now review the key ideas used in both [2] and the present paper. We begin by introducing the special functions $f_{\alpha}(x)$ and $\bar{f}_{\alpha}(x)$ in $C_{\alpha}$ defined by

$$
f_{\alpha}(x)=\sum_{j=1}^{\infty} \frac{\sin 2 \pi j x}{j^{\alpha}} \quad \text { and } \quad \bar{f}_{\alpha}(x)=\sum_{j=1}^{\infty} \frac{\cos 2 \pi j x}{j^{\alpha}} .
$$

Informally speaking, these functions are extremal in $C_{\alpha}$ in the sense that their Fourier coefficients are of maximal size. Furthermore, all Fourier coefficients are positive, which makes it relatively easy to obtain lower bounds for $L^{2}$ norms of sums of dilated functions.

When $\alpha=1$, the first series in (19) is the Fourier series of the function

$$
f_{1}(x)=\pi(1 / 2-\{x\}),
$$

where $\{\cdot\}$ denotes fractional part. This means that, up to multiplication by a constant, $f_{1}$ is the first Bernoulli polynomial on $[0,1]$, extended with period one. Convergence problems for (3) and (5) have been investigated extensively for $f=f_{1}$, starting probably with Riemann's $\mathrm{Ha}$ bilitationsschrift of 1852. Such series have been called Davenport series in honor of Harold Davenport, who was the first to study them in this general form [12]. See [17] for a survey on the history of the subject and several results on the convergence problem for series involving this function. Convergence problems for Davenport series have an interesting connection with fractal geometry, see for example [18].

The convergence problem for series involving the function $f_{1}$ is connected with sums involving greatest common divisors through the formula

$$
\int_{0}^{1}(\{k x\}-1 / 2)(\{\ell x\}-1 / 2) d x=\frac{1}{12} \frac{(\operatorname{gcd}(k, \ell))^{2}}{k \ell}
$$


for positive integers $k, \ell$, which was first stated by Franel and formally proved by Landau in 1924. Consequently we have

$$
\int_{0}^{1}\left(\sum_{k=1}^{N} c_{k} f_{1}\left(n_{k} x\right)\right)^{2} d x=\frac{\pi^{2}}{12} \sum_{k, \ell=1}^{N} c_{k} c_{\ell} \frac{\left(\operatorname{gcd}\left(n_{k}, n_{\ell}\right)\right)^{2}}{n_{k} n_{\ell}}
$$

But much more is true since the Fourier coefficients of $f_{1}$ are positive and maximal: By an observation of Koksma [21] we have

$$
\int_{0}^{1}\left(\sum_{k=1}^{N} c_{k} f\left(n_{k} x\right)\right)^{2} d x \ll \sum_{k, \ell=1}^{N}\left|c_{k} c_{\ell}\right| \frac{\left(\operatorname{gcd}\left(n_{k}, n_{\ell}\right)\right)^{2}}{n_{k} n_{\ell}}
$$

for every function $f$ in $C_{1}$.

The relation between $L^{2}$ norms of sums of dilated functions and sums involving greatest common divisors extends to the classes $C_{\alpha}$ for $1 / 2<\alpha<1$. This was first observed by Mikolás [26], who proved that for the Hurwitz zeta function $\zeta(1-\alpha, \cdot)$ we have

$$
\int_{0}^{1} \zeta(1-\alpha,\{k x\}) \zeta(1-\alpha,\{\ell x\}) d x=2 \Gamma(\alpha)^{2} \frac{\zeta(2 \alpha)}{(2 \pi)^{2 \alpha}} \frac{(\operatorname{gcd}(k, \ell))^{2 \alpha}}{(k \ell)^{\alpha}}
$$

for positive integers $k, \ell$ and for $\alpha>1 / 2$. Hurwitz's formula states that for $\alpha>1$ and $x \in[0,1]$ we have

$$
\zeta(1-\alpha, x)=\frac{\Gamma(\alpha)}{(2 \pi)^{\alpha}}\left(e^{-\pi i \alpha / 2}\left(\sum_{j=1}^{\infty} \frac{e^{2 \pi i j x}}{j^{\alpha}}\right)+e^{\pi i \alpha / 2}\left(\sum_{j=1}^{\infty} \frac{e^{-2 \pi i j x}}{j^{\alpha}}\right)\right)
$$

(see for example [20] for a simple proof), which implies that

$$
\zeta(1-\alpha, x)=\frac{2 \Gamma(\alpha)}{(2 \pi)^{\alpha}}\left(\cos (\pi \alpha / 2)\left(\sum_{j=1}^{\infty} \frac{\cos 2 \pi j x}{j^{\alpha}}\right)+\sin (\pi \alpha / 2)\left(\sum_{j=1}^{\infty} \frac{\sin 2 \pi j x}{j^{\alpha}}\right)\right) .
$$

Thus $\zeta(1-\alpha, x)$ is a function whose Fourier coefficients are precisely of asymptotic order $j^{-\alpha}$, and in particular $\zeta(1-\alpha, x) \in C_{\alpha}$. As Mikolás showed, (24) continues to hold for $\alpha>1 / 2$ and $0<x<1$, which leads to (23) by the orthogonality of the trigonometric system. By the same argument as for the case $\alpha=1$, we get that

$$
\int_{0}^{1}\left(\sum_{k=1}^{N} c_{k} f\left(n_{k} x\right)\right)^{2} d x \ll \sum_{k, \ell=1}^{N}\left|c_{k} c_{\ell}\right| \frac{\left(\operatorname{gcd}\left(n_{k}, n_{\ell}\right)\right)^{2 \alpha}}{\left(n_{k} n_{\ell}\right)^{\alpha}}
$$

for every function $f$ in $C_{\alpha}$ (see Lemma 1 below). For the special function $f_{\alpha}(x)$ from (19) we get

$$
\int_{0}^{1}\left(\sum_{k=1}^{N} c_{k} f_{\alpha}\left(n_{k} x\right)\right)^{2} d x=\frac{\zeta(2 \alpha)}{2} \sum_{k, \ell=1}^{N} c_{k} c_{\ell} \frac{\left(\operatorname{gcd}\left(n_{k}, n_{\ell}\right)\right)^{2 \alpha}}{\left(n_{k} n_{\ell}\right)^{\alpha}},
$$

as will also be established in Lemma 1 below. 
Our two estimates (22) and (25), as well as the two identities (21) and (26), show that to understand the convergence of (3) and (5) for $f$ in $C_{\alpha}$ it is important to have good upper and lower bounds for sums of the form

$$
\sum_{k, \ell=1}^{N} c_{k} c_{\ell} \frac{(\operatorname{gcd}(k, \ell))^{2 \alpha}}{(k \ell)^{\alpha}} \quad \text { and } \quad \sum_{k, \ell=1}^{N} c_{k} c_{\ell} \frac{\left(\operatorname{gcd}\left(n_{k}, n_{\ell}\right)\right)^{2 \alpha}}{\left(n_{k} n_{\ell}\right)^{\alpha}} .
$$

Now let $G_{N}^{(\alpha)}$ be the $N \times N$ matrix with entries $g_{k \ell}$ given by

$$
g_{k \ell}=\frac{(\operatorname{gcd}(k, \ell))^{2 \alpha}}{(k \ell)^{\alpha}}
$$

and $H_{N}^{(\alpha)}$ the $N \times N$ matrix with entries $h_{k \ell}$ of the form

$$
h_{k \ell}=\frac{\left(\operatorname{gcd}\left(n_{k}, n_{\ell}\right)\right)^{2 \alpha}}{\left(n_{k} n_{\ell}\right)^{\alpha}} .
$$

It is a well-known fact that both these matrices are positive definite (see e.g. [24]). Thus for the largest eigenvalue $\Lambda\left(G_{N}^{(\alpha)}\right)$ of $G_{N}^{(\alpha)}$ we have

$$
\Lambda\left(G_{N}^{(\alpha)}\right)=\max _{\substack{c_{1}, \ldots, c_{N}: \\ c_{1}^{2}+\cdots+c_{N}^{2}=1}} \sum_{k, \ell=1}^{N} c_{k} c_{\ell} \frac{(\operatorname{gcd}(k, \ell))^{2 \alpha}}{(k \ell)^{\alpha}},
$$

and for the largest eigenvalue $\Lambda\left(H_{N}^{(\alpha)}\right)$ of $H_{N}^{(\alpha)}$ we have

$$
\Lambda\left(H_{N}^{(\alpha)}\right)=\max _{\substack{c_{1}, \ldots, c_{N}: \\ c_{1}^{2}+\cdots+c_{N}^{2}=1}} \sum_{k, \ell=1}^{N} c_{k} c_{\ell} \frac{\left(\operatorname{gcd}\left(n_{k}, n_{\ell}\right)\right)^{2 \alpha}}{\left(n_{k} n_{\ell}\right)^{\alpha}} .
$$

Consequently, by (25) and (26), the problem of finding upper and lower bounds for the largest eigenvalue (or the square-root of the spectral norm) of $G_{N}^{(\alpha)}$ and $H_{N}^{(\alpha)}$ is precisely the same as that of finding general upper bounds for respectively

$$
\int_{0}^{1}\left(\sum_{k=1}^{N} c_{k} f(k x)\right)^{2} d x \quad \text { and } \quad \int_{0}^{1}\left(\sum_{k=1}^{N} c_{k} f\left(n_{k} x\right)\right)^{2} d x
$$

when $f$ is in $C_{\alpha}$, and for finding lower bounds for these integrals in the special case when $f=f_{\alpha}$.

The problem of calculating the largest eigenvalue $\Lambda\left(G_{N}^{(\alpha)}\right)$ of $G_{N}^{(\alpha)}$, and accordingly the problem of estimating the integral on the left-hand side of (30), was solved by Hilberdink [16], who proved that

$$
\Lambda\left(G_{N}^{(\alpha)}\right)=\frac{1}{\zeta(2)}\left(e^{\gamma} \log \log N+\mathscr{O}(1)\right)^{2} \quad \text { for } \quad \alpha=1
$$

and

$$
\Lambda\left(G_{N}^{(\alpha)}\right) \ll \exp \left(K \frac{(\log N)^{1-\beta}}{\log \log N}\right) \quad \text { for } \quad \frac{1}{2}<\alpha<1
$$


In (32) the constants $K$ depends on $\alpha$, and (32) is optimal except for the precise value of $K$. For $H_{N}^{(\alpha)}$, in Lemma 4 and Theorem 5 of [2] it was shown that

$$
\Lambda\left(H_{N}^{(\alpha)}\right) \ll(\log \log N)^{4} \quad \text { for } \quad \alpha=1
$$

and

$$
\Lambda\left(H_{N}^{(\alpha)}\right) \ll \exp \left(K \frac{(\log N)^{1-\alpha}}{(\log \log N)^{\alpha}}\right) \quad \text { for } \quad \frac{1}{2}<\alpha<1,
$$

where the constant $K$ depends on $\alpha$. Here (34) is optimal except for the precise value of the constant $K$, but it remains a profound problem to decide whether the exponent 4 of $\log \log N$ on the right-hand side of (33) is optimal. By a classical theorem of Gál [13], it is known that this exponent can not be smaller than 2 .

As noted, the results (31)-(34) imply corresponding upper bounds for the integrals in (30) when $f \in C_{\alpha}$, and the optimality of (31), (32) and (34) implies corresponding lower bounds for the integrals in (30) in the special case when $f=f_{\alpha}$; this is the reason why the exponential factor from (32) appears and Theorem 1, and that from (34) appears in Theorem 2. When comparing the bounds for the largest eigenvalues of $G_{N}^{(\alpha)}$ and $H_{N}^{(\alpha)}$, respectively, we note that in the case $\alpha=1$ there is an additional factor $(\log \log N)^{2}$ in (33) as compared with (31). However, as mentioned above, this extra factor possibly can be avoided since we do not know whether (33) is optimal. In the case $1 / 2<\alpha<1$ there is a difference between the denominator in the exponential terms in (32) and (34), respectively, which is $\log \log N$ in the one case and $(\log \log N)^{\alpha}$ in the other case. Since both results are optimal, this shows that there really is a significant difference between the spectral norms of $G_{N}^{(\alpha)}$ and of $H_{N}^{(\alpha)}$, and accordingly also a difference between the convergence problems for (3) and (5). In [16], a connection is established between the spectral norm of $G_{N}^{(\alpha)}$ and the maximal order of magnitude of the Riemann zeta-function along vertical lines, using Soundararajan's "resonance method" from [29]. However, Hilberdink's results cannot reach the stronger lower bounds of Montgomery [27], which in turn bear a striking resemblance to the bounds for the spectral norm of $H_{N}^{(\alpha)}$ in [2]. This is something which we cannot explain yet, and which calls for further research.

We close this section by making an observation on our extremal functions $f_{\alpha}$ and $\bar{f}_{\alpha}$ in (19) that will be needed in the sequel. We note first that they are, up to normalization, the even and odd parts of the Hurwitz zeta function. In fact, from the Fourier series representation in (24) it is easily seen that

$$
\frac{\zeta(1-\alpha, x)-\zeta(1-\alpha, 1-x)}{2}=\frac{2 \Gamma(\alpha)}{(2 \pi)^{\alpha}} \sin (\pi \alpha / 2) f_{\alpha}(x)
$$

and

$$
\frac{\zeta(1-\alpha, x)+\zeta(1-\alpha, 1-x)}{2}=\frac{2 \Gamma(\alpha)}{(2 \pi)^{\alpha}} \cos (\pi \alpha / 2) \bar{f}_{\alpha}(x)
$$


These representations can be used to describe the rate with which $f_{\alpha}(x)$ and $\bar{f}_{\alpha}(x)$ tend to infinity as $x \rightarrow 0$. Mikolás proved that for fixed $\alpha \in(1 / 2,1)$ we have

$$
\lim _{x \rightarrow 0+} x^{1-\alpha} \zeta(1-\alpha, x)=1
$$

(this is equation (12) in [26]). Consequently, since $\lim _{x \rightarrow 0+} \zeta(1-\alpha, 1-x)=\zeta(1-\alpha, 1)=\zeta(1-\alpha)$ is a constant, we have

$$
\lim _{x \rightarrow 0+} x^{1-\alpha} f_{\alpha}(x)=\frac{(2 \pi)^{\alpha}}{\Gamma(\alpha) \sin (\pi \alpha / 2)} .
$$

In particular this implies that

$$
f_{\alpha} \in L^{p}(0,1) \quad \text { for } \quad p<\frac{1}{1-\alpha},
$$

which will be a crucial ingredient in the proof of the necessary condition for almost everywhere convergence of (5). More precisely, (37) implies that for any $\alpha \in(1 / 2,1)$ the function $f_{\alpha}$ is in $L^{2+\delta}$ for some $\delta=\delta(\alpha)>0$, which will allow us to apply Lyapunov's central limit theorem (which requires the existence of an absolute moment of order $2+\delta$ for some $\delta>0$ ). Similar results hold if $f_{\alpha}$ is replaced by $\bar{f}_{\alpha}$.

\section{AuXiliary Results}

In the sequel, we use the notation $\|\cdot\|$ for the $L^{2}(0,1)$ norm. Throughout the rest of this paper, we will always assume that $\alpha \in(1 / 2,1)$.

Lemma 1. Assume that $f \in C_{\alpha}$. Then

$$
\int_{0}^{1}\left(\sum_{k=1}^{N} c_{k} f\left(n_{k} x\right)\right)^{2} d x \ll \sum_{k=1}^{N}\left|c_{k} c_{\ell}\right| \frac{\left(\operatorname{gcd}\left(n_{k}, n_{\ell}\right)\right)^{2 \alpha}}{\left(n_{k} n_{\ell}\right)^{\alpha}} .
$$

For the particular function $f_{\alpha}$ from (19) we have

$$
\int_{0}^{1}\left(\sum_{k=1}^{N} c_{k} f_{\alpha}\left(n_{k} x\right)\right)^{2} d x=\frac{\zeta(2 \alpha)}{2} \sum_{k, \ell=1}^{N} c_{k} c_{\ell} \frac{\left(\operatorname{gcd}\left(n_{k}, n_{\ell}\right)\right)^{2 \alpha}}{\left(n_{k} n_{\ell}\right)^{\alpha}} .
$$

Note that as a special case of Lemma 1 we have

$$
\int_{0}^{1}\left(\sum_{k=1}^{N} c_{k} f(k x)\right)^{2} d x \ll \sum_{k, \ell=1}^{N}\left|c_{k} c_{\ell}\right| \frac{(\operatorname{gcd}(k, \ell))^{2 \alpha}}{(k \ell)^{\alpha}} .
$$

Proof of Lemma 1. The argument needed for the proof of Lemma 1 is a simple generalization of the arguments leading to (21) and (22), respectively. We write

$$
f(x) \sim \sum_{j=1}^{\infty} a_{j} \sin 2 \pi j x,
$$


assuming, to shorten formulas, that $f$ is an odd function; the proof in the general case is exactly the same. Then, by the orthogonality of the trigonometrical system, for arbitrary positive integers $m, n$ we have

$$
\begin{aligned}
\int_{0}^{1} f(m x) f(n x) d x & =\frac{1}{2} \sum_{j_{1}, j_{2}=1}^{\infty} a_{j_{1}} a_{j_{2}} 1\left(j_{1} m=j_{2} n\right) \\
& =\frac{1}{2} \sum_{j=1}^{\infty} a_{j m / \operatorname{gcd}(m, n)} a_{j n / \operatorname{gcd}(m, n)} \\
& \ll \sum_{j=1}^{\infty}\left(\frac{\operatorname{gcd}(m, n)}{j m}\right)^{\alpha}\left(\frac{\operatorname{gcd}(m, n)}{j n}\right)^{\alpha} \\
& \ll\left(\frac{(\operatorname{gcd}(m, n))^{2}}{m n}\right)^{\alpha} .
\end{aligned}
$$

In (40), we used the fact that $j_{1} m=j_{2} n$ holds if and only if $j_{1}=j n / \operatorname{gcd}(m, n)$ and $j_{2}=$ $j m / \operatorname{gcd}(m, n)$ for some positive integer $j$. Applying this inequality for all pairs $\left(n_{k}, n_{\ell}\right)$ gives the first part of the lemma.

In the case $f=f_{\alpha}$ we have $a_{j}=j^{-\alpha}, j \geq 1$. Inserting this into (41) we get

$$
\int_{0}^{1} f(m x) f(n x) d x=\frac{1}{2} \sum_{j=1}^{\infty}\left(\frac{\operatorname{gcd}(m, n)}{j m}\right)^{\alpha}\left(\frac{\operatorname{gcd}(m, n)}{j n}\right)^{\alpha}=\frac{\zeta(2 \alpha)}{2}\left(\frac{(\operatorname{gcd}(m, n))^{2}}{m n}\right)^{\alpha} .
$$

Again we obtain the desired result by summing over all pairs $\left(n_{k}, n_{\ell}\right)$.

Lemma 2. Assume that $f \in C_{\alpha}$. There exist constants $K_{1}, K_{2}$ such that

$$
\left\|\sum_{k=1}^{N} c_{k} f(k x)\right\|^{2} \ll \exp \left(\frac{K_{1}(\log N)^{1-\alpha}}{\log \log N}\right) \sum_{k=1}^{N} c_{k}^{2}
$$

and

$$
\left\|\sum_{k=1}^{N} c_{k} f\left(n_{k} x\right)\right\|^{2} \ll \exp \left(\frac{K_{2}(\log N)^{1-\alpha}}{(\log \log N)^{\alpha}}\right) \sum_{k=1}^{N} c_{k}^{2} .
$$

We can choose $K_{1}, K_{2}$ such that

$$
K_{1}<3 /(1-\alpha)+4 / \sqrt{2 \alpha-1} \quad \text { and } \quad K_{2}<6 /(1-\alpha)+7\left(|\log (2 \alpha-1)|^{1 / 2}+1\right) .
$$

By Lemma 1 and (28) and (29), the estimates in Lemma 2 follow from corresponding upper bounds for the largest eigenvalues of the matrices $G_{N}^{(\alpha)}$ and $H_{N}^{(\alpha)}$, respectively, which were already stated in (32) and (34). The given value for $K_{1}$ is a coarse estimate for that given in a more precise form in the proof of [16, Theorem 2.3] and at the end of [16, Section 3]; the value for $K_{2}$ is obtained by using the sharpening of the arguments from [2] found in the recent paper [8].

Using the same method as in the proof of the Rademacher-Menshov inequality, we easily obtain the following lemma, which is a maximal version of Lemma 2. Note that the proof of 
the Rademacher-Menshov inequality gives an additional logarithmic factor, which however in our case can be included in the exponential term if we slightly increase the value of the constants.

Lemma 3. Assume that $f \in C_{\alpha}$. Then there exist constants $K_{1}, K_{2}$ such that

$$
\| \max _{1 \leq M \leq N}\left|\sum_{k=1}^{M} c_{k} f(k x)\right|||^{2} \ll \exp \left(\frac{K_{1}(\log N)^{1-\alpha}}{\log \log N}\right) \sum_{k=1}^{N} c_{k}^{2}
$$

and

We can choose $K_{1}, K_{2}$ such that

$$
\left\|\max _{1 \leq M \leq N}\left|\sum_{k=1}^{M} c_{k} f\left(n_{k} x\right)\right|\right\|^{2} \ll \exp \left(\frac{K_{2}(\log N)^{1-\alpha}}{(\log \log N)^{\alpha}}\right) \sum_{k=1}^{N} c_{k}^{2} .
$$

$$
K_{1}<3 /(1-\alpha)+4 / \sqrt{2 \alpha-1} \quad \text { and } \quad K_{2}<6 /(1-\alpha)+7\left(|\log (2 \alpha-1)|^{1 / 2}+1\right) .
$$

Lemma 4 ([1, Lemma 6]). Assume that for every given $\varepsilon>0$ there exists an $M_{0}(\varepsilon)$ such that

$$
\left\|\sup _{M>M_{0}}\left|\sum_{k=M_{0}+1}^{M} c_{k} f(k x)\right|\right\| \leq \varepsilon .
$$

Then

$$
\sum_{k=1}^{\infty} c_{k} f(k x)
$$

is almost everywhere convergent.

For the formulation of the following lemma we note that the unit interval, equipped with Borel sets and Lebesgue measure is a probability space. Throughout the rest of this paper, we will use the symbols $\mathbb{P}$ and $\mathbb{E}$ with respect to this probability space. The following lemma is a variant of [2, Lemma 5]. We write $\log _{2}$ for the dyadic logarithm.

Lemma 5. For given $\alpha \in(1 / 2,1)$, let $\eta=12 /(2 \alpha-1)$ and let $1 \leq S_{1}<T_{1}<S_{2}<T_{2}<\ldots$ be integers such that

$$
S_{i+1} \geq T_{i}+\eta \log _{2} i .
$$

Furthermore, let $\Delta_{1}, \Delta_{2}, \ldots$ be sets of integers such that $\Delta_{i} \subset\left[2^{S_{i}}, 2^{T_{i}}\right]$ and each element of $\Delta_{i}$ is divisible by $2^{S_{i}}$. For $i \geq 1$ and $x \in(0,1)$ set

$$
X_{i}=X_{i}(x):=\sum_{k \in \Delta_{i}} f_{\alpha}(k x) .
$$

Then there exist independent random variables $Y_{1}, Y_{2}, \ldots$ on the probability space $((0,1), \mathscr{B}, \mathbb{P})$ such that $\mathbb{E} Y_{i}=0$ and

$$
\left\|X_{i}-Y_{i}\right\| \ll i^{-2} \cdot \# \Delta_{i} .
$$

For the proof of Lemma 5, we need the following lemma, which is [3, Lemma 3.1]. Here, given an integrable function $g(x)$ on $[0,1]$ and an arbitrary integer $m$, we write $[g]_{m}$ for the function which takes the constant value

$$
m \int_{k / m}^{(k+1) / m} g(x) d x
$$


in the intervals $[k / m,(k+1) / m)$, for $k=0, \ldots, m-1$.

Lemma 6 ([3, Lemma 3.1]). Assume that $f \in C_{\alpha}$. Let $k \geq 1$ be a positive integer, and write $g(x)=f(k x)$. Then for any integer $m \geq k$ we have

$$
\left\|g-[g]_{m}\right\| \ll\left(\frac{k}{m}\right)^{(2 \alpha-1) / 6}
$$

Proof of Lemma 5: Let $\mathscr{F}_{i}$ denote the $\sigma$-field generated by the dyadic intervals

$$
U_{j}:=\left[j 2^{-S_{i+1}},(j+1) 2^{-S_{i+1}}\right), \quad 0 \leq j<2^{S_{i+1}},
$$

and set

$$
\xi_{k}=\xi_{k}(\cdot)=\mathbb{E}\left(f_{\alpha}(k \cdot) \mid \mathscr{F}_{i}\right), \quad k \in \Delta_{i}
$$

and

$$
Y_{i}=Y_{i}(x)=\sum_{k \in \Delta_{i}} \xi_{k}(x)
$$

Then we clearly have $\mathbb{E} \xi_{k}=0$, which implies $\mathbb{E} Y_{i}=0$. By Lemma 6 and (42) we have for every $k \in \Delta_{i}$

$$
\left\|\xi_{k}(\cdot)-f_{\alpha}(k \cdot)\right\| \ll\left(\frac{k}{2^{S_{i+1}}}\right)^{(2 \alpha-1) / 6} \ll\left(\frac{2^{T_{i}}}{2^{T_{i}+\eta \log _{2} i}}\right)^{(2 \alpha-1) / 6} \ll i^{-\eta(2 \alpha-1) / 6} \ll i^{-2},
$$

which implies that

$$
\left\|X_{i}-Y_{i}\right\| \ll i^{-2} \cdot \# \Delta_{i}
$$

Since by assumption every $k \in \Delta_{i+1}$ is a multiple of $2^{S_{i+1}}$, each interval $U_{j}$ in (43) is a period interval of $f_{\alpha}(k x)$ for all $k \in \Delta_{i+1}$, and consequently also for $\xi_{k}$ for all $k \in \Delta_{i+1}$. Consequently $Y_{i+1}$ is independent of the $\sigma$-field $\mathscr{F}_{i}$. Since $\mathscr{F}_{1} \subset \mathscr{F}_{2} \subset \ldots$ and since $Y_{i}$ is $\mathscr{F}_{i}$-measurable, the random variables $Y_{1}, Y_{2}, \ldots$ are independent.

The following lemma is a simple consequence of [16, Proposition 3.1], from which it can be deduced in the same way as relation (3.2) of [16].

Lemma 7. We have

$$
\sum_{k, \ell \leq N} c_{k} c_{\ell} \frac{(\operatorname{gcd}(k, \ell))^{2 \alpha}}{(k \ell)^{\alpha}} \leq \sum_{k \leq N^{2}} b_{k}^{2}
$$

where $b_{k}$ are defined by

$$
b_{k}=\frac{1}{k^{\alpha}} \sum_{d \mid k} d^{\alpha}\left|c_{d}\right|
$$




\section{ProOFs}

Proof of the convergence part of Theorem 1: Throughout this proof, we will write $K_{1}$ for the constant in the statement of Theorem 1 , and $K_{2}$ for the constant in the statement of the first part of Lemma 2. Note that we can assume that $K_{1}>K_{2}$. Relation (6) implies that

$$
\sum_{k=e^{m}+1}^{e^{m+1}} c_{k}^{2} \exp \left(\frac{K_{1}(\log k)^{1-\alpha}}{\log \log k}\right) \ll 1 \quad \text { for } m \geq 1,
$$

which also implies that

$$
\sum_{k=e^{m}+1}^{e^{m+1}} c_{k}^{2} \ll \exp \left(\frac{-K_{1} m^{1-\alpha}}{\log m}\right) \quad \text { for } m \geq 1 .
$$

Consequently by Lemma 2 we have, for any $M, N$ satisfying $e^{m}<M<N<e^{m+1}$,

$$
\begin{aligned}
\left\|\sum_{k=M}^{N} c_{k} f(k x)\right\|^{2} & \ll \exp \left(\frac{K_{2}(m+1)^{1-\alpha}}{\log (m+1)}\right) \exp \left(\frac{-K_{1} m^{1-\alpha}}{\log m}\right) \\
& \ll \exp \left(\frac{-\varepsilon m^{1-\alpha}}{\log m}\right)
\end{aligned}
$$

for some $\varepsilon>0$, since $K_{1}>K_{2}$. For given $M<N$, let $\hat{m}$ denote the integer for which $M \in$ $\left(e^{\hat{m}}, e^{\hat{m}+1}\right]$, and $\hat{n}$ the integer for which $N \in\left(e^{\hat{n}}, e^{\hat{n}+1}\right]$. If $\hat{m}=\hat{n}$, then by (44) we have

$$
\left\|\sum_{k=M}^{N} c_{k} f(k x)\right\| \ll \exp \left(\frac{-\varepsilon \hat{m}^{1-\alpha}}{\log \hat{m}}\right) .
$$

If $\hat{m}<\hat{n}$, then by (44) and Minkowski's inequality we have

$$
\begin{aligned}
& \left\|\sum_{k=M}^{N} c_{k} f(k x)\right\| \\
\ll & \left\|\sum_{k=M}^{\hat{m}+1} c_{k} f(k x)\right\|+\sum_{m=\hat{m}+1}\left\|\sum_{k=e^{m}+1}^{e^{m+1}} c_{k} f(k x)\right\|+\left\|\sum_{k=e^{\hat{n}}+1}^{N} c_{k} f(k x)\right\| \\
\ll & \sum_{m=\hat{m}}^{\infty} \exp \left(\frac{-\varepsilon m^{1-\alpha}}{2 \log m}\right) .
\end{aligned}
$$

Both (45) and (46) can be made arbitrarily small if $\hat{m}$ is assumed to be sufficiently large (note that (46) is the tail of a convergent series). Thus by the Cauchy convergence test the series $\sum_{k=1}^{\infty} c_{k} f(k x)$ is convergent in $L^{2}$. In a similar way, using Lemma 3 instead of Lemma 2, we obtain for any $M<N$

$$
\| \max _{M<L \leq N}\left|\sum_{k=M}^{L} c_{k} f(k x)\right| \mid \ll \sum_{m=\hat{m}}^{\infty} \exp \left(\frac{-\varepsilon m^{1-\alpha}}{2 \log m}\right),
$$

where $\hat{m}$ is defined as before. Again the right-hand side can be made arbitrarily small if $M$ is assumed to be sufficiently large. Thus the monotone convergence theorem and Lemma 4 
imply that the series $\sum_{k=1}^{\infty} c_{k} f(k x)$ is almost everywhere convergent.

Proof of the optimality of Theorem 1: For given $\alpha \in(1 / 2,1)$, we will show that there exists a sequence $\left(c_{k}\right)_{k \geq 1}$ satisfying (6) for a "small" value of $K$, for which for the function $f(x)=f_{\alpha}(x)$ from (19) the series $\sum_{k=1}^{\infty} c_{k} f_{\alpha}(k x)$ is divergent in $L^{2}$. We will construct $\left(c_{k}\right)_{k \geq 1}$ such that it is supported on a set of indices which have a small number of prime factors; this idea already appears in $[2,13,16]$ and other places. However, there it is only used to construct a finite sequence, whereas in the present case we have to construct an infinite sequence. Note that by (23), (35) and (36) the $L^{2}$ norm of sums of dilated functions $f_{\alpha}(x), \bar{f}_{\alpha}(x)$ and $\zeta(1-\alpha, x)$ is the same, up to multiplication with a constant, and consequently we could also use the functions $\bar{f}_{\alpha}$ or $\zeta(1-\alpha, x)$ instead of $f_{\alpha}(x)$.

We write $\left(p_{r}\right)_{r \geq 1}$ for the sequences of primes in increasing order. We define sets $\Delta_{i}$ in the following way: for given $i \geq 1$, the set $\Delta_{i}$ contains those positive integers which are of the form

$$
2^{2 i} p_{1}^{w_{1}} p_{2}^{w_{2}} \ldots p_{i}^{w_{i}} \quad \text { for } \quad\left(w_{1}, \ldots, w_{i}\right) \in\{0,1\}^{i} .
$$

By construction the sets $\Delta_{i}, i \geq 1$, are disjoint (since all numbers in $\Delta_{i}$ are multiples of either $2^{2 i}$ or $2^{2 i+1}$, but not of $2^{2 i+2}$ ). Note that the number of elements of $\Delta_{i}$ is $2^{i}$.

Let $\varepsilon>0$ be fixed, and set $\eta=(1-2 \varepsilon) /(1+\varepsilon)$. We define

$$
c_{k}= \begin{cases}2^{-i / 2} i^{-1} \exp \left(-\frac{\eta}{2(1-\alpha)}(\log k)^{1-\alpha}(\log \log k)^{-1}\right) & \text { if } k \in \Delta_{i} \text { for some } i \geq 1, \\ 0 & \text { otherwise. }\end{cases}
$$

Then we have

$$
\begin{aligned}
\sum_{k=1}^{\infty} c_{k}^{2} \exp \left(\frac{\eta}{1-\alpha}(\log k)^{1-\alpha}(\log \log k)^{-1}\right) & =\sum_{i=1}^{\infty} \sum_{k \in \Delta_{i}} 2^{-i} i^{-2} \\
& =\sum_{i=1}^{\infty} i^{-2}<\infty
\end{aligned}
$$

By the prime number theorem for all sufficiently large $i$ for all $k \in \Delta_{i}$ we have

$$
k \leq 2^{2 i} \prod_{r=1}^{i} p_{r} \leq 2^{2 i}\left(((1+\varepsilon) i \log i)^{i}\right),
$$

and consequently for sufficiently large $i$ and for all $k \in \Delta_{i}$

$$
\frac{(\log k)^{1-\alpha}}{(\log \log k)} \leq(1+\varepsilon)(i \log i)^{1-\alpha}(\log i)^{-1}=(1+\varepsilon) i^{1-\alpha}(\log i)^{-\alpha} .
$$

Thus for $i \geq 1$ for all $k \in \Delta_{i}$ we have

$$
c_{k} \gg 2^{-i / 2} i^{-1} \exp \left(-\frac{\eta(1+\varepsilon)}{2(1-\alpha)} i^{1-\alpha}(\log i)^{-\alpha}\right) .
$$


Using the second part of Lemma 1 and the facts that $f_{\alpha}$ has only positive Fourier coefficients and that all coefficients $c_{k}$ are non-negative, we have

$$
\begin{aligned}
\lim _{N \rightarrow \infty}\left\|\sum_{k=1}^{N} c_{k} f_{\alpha}(k x)\right\|^{2} & \geq \lim _{M \rightarrow \infty}\left\|\sum_{i=1}^{M} \sum_{k \in \Delta_{i}} c_{k} f_{\alpha}(k x)\right\|^{2} \\
& \geq \lim _{M \rightarrow \infty} \sum_{i=1}^{M}\left\|\sum_{k \in \Delta_{i}} c_{k} f_{\alpha}(k x)\right\|^{2} \\
& =\sum_{i=1}^{\infty} \sum_{k, \ell \in \Delta_{i}} c_{k} c_{\ell} \frac{(\operatorname{gcd}(k, \ell))^{2 \alpha}}{(k \ell)^{\alpha}} .
\end{aligned}
$$

By the structure of the set $\Delta_{i}$ for any fixed $k \in \Delta_{i}$ we have

$$
\sum_{\ell \in \Delta_{i}} \frac{(\operatorname{gcd}(k, \ell))^{2 \alpha}}{(k \ell)^{\alpha}}=\prod_{r=1}^{i}\left(1+p_{r}^{-\alpha}\right)
$$

which implies

$$
\sum_{k, \ell \in \Delta_{i}} \frac{(\operatorname{gcd}(k, \ell))^{2 \alpha}}{(k \ell)^{\alpha}}=2^{i} \prod_{r=1}^{i}\left(1+p_{r}^{-\alpha}\right)
$$

(an argument of this type already appears in Gál's paper [13]). By the prime number theorem we have

$$
\prod_{r=1}^{i}\left(1+p_{r}^{-\alpha}\right) \gg \exp \left(\frac{1-\varepsilon}{1-\alpha} i^{1-\alpha}(\log i)^{-\alpha}\right)
$$

Combining (47), (48) and (49) we get

$$
\lim _{N \rightarrow \infty}\left\|\sum_{k=1}^{N} c_{k} f_{\alpha}(k x)\right\|^{2} \gg \sum_{i=1}^{\infty} i^{-2} \exp \left(\frac{(1-\varepsilon)-\eta(1+\varepsilon)}{1-\alpha} i^{1-\alpha}(\log i)^{-\alpha}\right) .
$$

Note that $(1-\varepsilon)-\eta(1+\varepsilon)=\varepsilon$, and thus the series on the right-hand side of (50) is divergent. Consequently the series $\sum_{k=1}^{\infty} c_{k} f_{\alpha}(k x)$ is divergent in $L^{2}$, although $\left(c_{k}\right)_{k \geq 1}$ satisfies the extra convergence condition (6) for $K=\eta /(1-\alpha)$. Note that by choosing $\varepsilon$ small, $\eta$ can be moved arbitrarily close to 1 . This proves the optimality of Theorem 1 , apart from the precise optimal value of the constant $K$ in (6).

Proof of the convergence part of Theorem 2: The proof of the convergence part of Theorem 2 can be given in exactly the same way as the proof of the convergence part of Theorem 1 above, using the second part of Lemma 2 and 3 instead of the first part, respectively.

Proof of the optimality of Theorem 2: The optimality of condition (7) in the case of $L^{2}$ convergence can be shown in a similar way as the optimality of condition (6) in Theorem 1. Again we construct a set of integers which is composed of a relatively small number of prime factors. In particular, again we will use an equality similar to (49), which allows a precise computation of the corresponding GCD sum. Again we choose $f=f_{\alpha}$, but as in the proof of the optimality of 
Theorem 1 we could also use the functions $\bar{f}_{\alpha}$ or $\zeta(1-\alpha, \cdot)$ instead. The main difference between the present case and the proof of Theorem 1 is the fact that we can make the sequence $\left(n_{k}\right)_{k \geq 1}$ grow as fast as we wish. Together with the well-established principle that lacunary sequences of functions show almost independent behavior, this is the reason why for Theorem 2 we can also prove optimality with respect to almost everywhere convergence (which was not possible for Theorem 1).

First we recall that $f_{\alpha} \in L^{p}(0,1)$ for $p<(1-\alpha)^{-1}$, which was established in (37). Thus we can choose $\delta \in(0,1)$ such that $2+\delta<(1-\alpha)^{-1}$. Furthermore, we can find a number $\beta \in(0,1)$ which satisfies

For this number $\beta$ we have

$$
\beta<\frac{\delta}{2+\delta}
$$

$$
\left(-\frac{1}{2}+\frac{\beta}{2}\right)(2+\delta)<-1 .
$$

Let $\left(p_{r}\right)_{r \geq 1}$ denote the sequence of primes in increasing order. We set $A(1)=1$ and

$$
A(i)=\left\lceil\beta \log _{2} i\right\rceil, \quad i \geq 2 .
$$

Here, and in the sequel, $\log _{2}$ denotes the logarithm in base 2 . We define the numbers $S_{i}$ and $T_{i}$ recursively in the following way:

- $S_{1}=2$,

- $T_{i}=S_{i}+\left\lceil\log _{2}\left(\Pi_{r=1}^{A(i)} p_{r}\right)\right\rceil, \quad i \geq 1$

- $S_{i+1}=T_{i}+\left\lceil\eta \log _{2} i\right\rceil, \quad i \geq 1, \quad$ where $\eta=12 /(2 \alpha-1)$.

Then obviously the numbers $\left(S_{i}\right)_{i \geq 1}$ and $\left(T_{i}\right)_{i \geq 1}$ satisfy the conditions of Lemma 5 . For $i \geq 1$, we define $\Delta_{i}$ as the set of all numbers $k$ of the form

$$
k=2^{S_{i}} \prod_{r=1}^{A(i)} p_{r}^{w_{r}}, \quad \text { where } \quad\left(w_{1}, \ldots, w_{A(i)}\right) \in\{0,1\}^{A(i)} .
$$

Then clearly all elements of $\Delta_{i}$ are divisible by $2^{S_{i}}$, and $\Delta_{i} \subset\left[2^{S_{i}}, 2^{T_{i}}\right]$; that is, the sets $\Delta_{i}$ also satisfy the assumptions of Lemma 5 . Let $\left(n_{k}\right)_{k \geq 1}$ denote the sequence consisting of the elements of $\bigcup_{i \geq 1} \Delta_{i}$, sorted in increasing order. Note that by definition we have

$$
\# \Delta_{i}=2^{A(i)} \in\left[i^{\beta}, 2 i^{\beta}\right] \text {. }
$$

Furthermore we define sets of integers $\Gamma_{i}, i \geq 1$, such that

$$
k \in \Gamma_{i} \quad \text { if and only if } \quad n_{k} \in \Delta_{i} .
$$

Then $\left(\Gamma_{i}\right)_{i \geq 1}$ is a decomposition of $\mathbb{N}$. Let $K_{1}$ denote a "small" constant with a value to be determined later. For every $k \geq 1$ there is an $i$ such that $k \in \Gamma_{i}$, and we define

$$
c_{k}=i^{-\beta / 2-1 / 2}(\log i)^{-1} \exp \left(-\frac{K_{1}(\log i)^{1-\alpha}}{2(\log \log i)^{\alpha}}\right) .
$$


Note that the value of $c_{k}$ only depends on the index $i$ for which $k \in \Gamma_{i}$. Thus we can also define numbers $\left(d_{i}\right)_{i \geq 1}$ such that

$$
d_{i}=c_{k} \quad \text { whenever } \quad k \in \Gamma_{i}, \quad \text { for } i \geq 1, k \geq 1,
$$

which implies that

$$
\sum_{k \in \Delta_{i}} c_{k} f_{\alpha}(k x)=d_{i} \sum_{k \in \Gamma_{i}} f_{\alpha}\left(n_{k} x\right)
$$

Furthermore we have

$$
\begin{aligned}
\sum_{k=1}^{\infty} c_{k}^{2} \exp \left(\frac{K_{1}(\log i)^{1-\alpha}}{(\log \log i)^{\alpha}}\right) & =\sum_{i \geq 1} \sum_{k \in \Gamma_{i}} i^{-\beta-1}(\log i)^{-2} \cdot \underbrace{\# \Gamma_{i}}_{\leq 2 i^{\beta}} \\
& \leq 2 \sum_{i \geq 1} i^{-1}(\log i)^{-2} .
\end{aligned}
$$

Since the series in (53) is convergent, the same holds for the series on the left-hand side of (52). Furthermore, since for $k \in \Gamma_{i}$ we have

$$
k \ll i^{\beta+1},
$$

the convergence of the left-hand side of (52) implies that there exists a positive constant $K_{2}$ (depending on $K_{1}$ ) such that

$$
\sum_{k=1}^{\infty} c_{k}^{2} \exp \left(K_{2}(\log k)^{1-\alpha}(\log \log k)^{-\alpha}\right)<\infty .
$$

As in the lines following (49) we get

$$
\begin{aligned}
\sum_{k, \ell \in \Gamma_{i}} \frac{\left(\operatorname{gcd}\left(n_{k}, n_{\ell}\right)\right)^{2 \alpha}}{\left(n_{k} n_{\ell}\right)^{\alpha}} & =\sum_{k, \ell \in \Delta_{i}} \frac{(\operatorname{gcd}(k, \ell))^{2 \alpha}}{(k \ell)^{\alpha}} \\
& =\# \Delta_{i} \prod_{r=1}^{A(i)}\left(1+p_{r}^{-\alpha}\right) \\
& \gg i^{\beta} \exp \left(K_{3}(\log i)^{1-\alpha}(\log \log i)^{-\alpha}\right)
\end{aligned}
$$

for some positive constant $K_{3}$. Together with the second part of Lemma 1 this implies

$$
\left\|\sum_{k \in \Gamma_{i}} c_{k} f_{\alpha}\left(n_{k} x\right)\right\|^{2} \gg i^{-1}(\log i)^{-2} \exp \left(\left(K_{3}-K_{1}\right)(\log i)^{1-\alpha}(\log \log i)^{-\alpha}\right) .
$$

Since all coefficients $\left(c_{k}\right)_{k \geq 1}$ are non-negative we have

$$
\lim _{N \rightarrow \infty}\left\|\sum_{k=1}^{N} c_{k} f_{\alpha}\left(n_{k} x\right)\right\|^{2} \geq \lim _{M \rightarrow \infty} \sum_{i=1}^{M}\left\|\sum_{k \in \Delta_{i}} c_{k} f_{\alpha}\left(n_{k} x\right)\right\|^{2} .
$$

Combining this with (55) we arrive at

$$
\lim _{N \rightarrow \infty}\left\|\sum_{k=1}^{N} c_{k} f_{\alpha}\left(n_{k} x\right)\right\|^{2} \gg \lim _{M \rightarrow \infty} \sum_{i=1}^{M} i^{-1}(\log i)^{-2} \exp \left(\left(K_{3}-K_{1}\right)(\log i)^{1-\alpha}(\log \log i)^{-\alpha}\right) .
$$


We can assume that $K_{1}$ was chosen so small that $K_{1}<K_{3}$. Then since the right-hand side of (56) is divergent, the series $\sum_{k=1}^{\infty} c_{k} f_{\alpha}\left(n_{k} x\right)$ is divergent in $L^{2}$. This proves the optimality of Theorem 2 for $L^{2}$ convergence (except for the exact value of the constant $K$ in the extra divergence condition).

To show that Theorem 2 is also optimal with respect to almost everywhere convergence, we apply Lemma 5 . As noted before, Lemma 5 can be used for $S_{i}, T_{i}, \Delta_{i}$ as defined above. Consequently there exist independent random variables $Y_{1}, Y_{2}, \ldots$ on $((0,1), \mathscr{B}, \mathbb{P})$ such that

$$
\left\|d_{i} Y_{i}-\sum_{k \in \Gamma_{i}} c_{k} f_{\alpha}\left(n_{k} x\right)\right\| \ll d_{i}\left\|Y_{i}-\sum_{k \in \Delta_{i}} f_{\alpha}(k x)\right\| \ll i^{-\beta / 2-1 / 2} i^{-2} \# \Delta_{i} \ll i^{-5 / 2+\beta / 2} .
$$

The proof of Lemma 5 shows that the random variables $Y_{i}$ are constructed as the conditional expectation of $\sum_{k \in \Delta_{i}} f_{\alpha}\left(n_{k} x\right)$ with respect to some appropriate $\sigma$-fields. Thus the conditional form of Jensen's inequality (see for example [23, Theorem 13.3]) implies that

$$
\mathbb{E}\left(\left|d_{i} Y_{i}\right|^{2+\delta}\right) \leq d_{i}^{2+\delta} \mathbb{E}\left(\left(\sum_{k \in \Gamma_{i}} f_{\alpha}\left(n_{k} \cdot\right)\right)^{2+\delta}\right) .
$$

We have chosen $\delta$ in such a way that $f_{\alpha} \in L^{2+\delta}(0,1)$. Thus by Minkowski's inequality we have

$$
\left\|\sum_{k \in \Gamma_{i}} f_{\alpha}\left(n_{k} \cdot\right)\right\|_{2+\delta} \leq\left\|f_{\alpha}\right\|_{2+\delta} \underbrace{\# \Gamma_{i}}_{=\# \Lambda_{i}} \ll i^{\beta},
$$

which together with (58) implies

$$
\mathbb{E}\left(\left|d_{i} Y_{i}\right|^{2+\delta}\right) \ll i^{(\beta / 2-1 / 2)(2+\delta)} .
$$

On the other hand, by (55) and (57) we have

$$
\mathbb{E}\left(\left(d_{i} Y_{i}\right)^{2}\right) \gg i^{-1}(\log i)^{-2} \exp \left(K_{4}(\log i)^{1-\alpha}(\log \log i)^{-\alpha}\right),
$$

where $K_{4}:=K_{3}-K_{1}$ is a positive constant (again we assume that $K_{1}$ was chosen sufficiently small). Let

$$
B_{M}=\sum_{i=1}^{M} \mathbb{E}\left(\left(d_{i} Y_{i}\right)^{2}\right), \quad D_{M}=\sum_{i=1}^{M} \mathbb{E}\left(\left|d_{i} Y_{i}\right|^{2+\delta}\right)
$$

and

$$
F_{M}(t)=\mathbb{P}\left(x \in(0,1): \sum_{i=1}^{M} d_{i} Y_{i}<t \sqrt{B_{M}}\right) .
$$

By (51) and (59) we see that the sequence $\left(D_{M}\right)_{M \geq 1}$ is bounded. On the other hand, by (60), we have

$$
B_{M} \gg \exp \left(K_{5}(\log M)^{1-\alpha}(\log \log M)^{-\alpha}\right)
$$


for some positive constant $K_{5}$, which in particular implies that $B_{M} \rightarrow \infty$ as $M \rightarrow \infty$. Thus, the so-called Lyapunov condition for the central limit theorem is satisfied, which implies that

$$
\sup _{t \in \mathbb{R}}\left|F_{M}(t)-\Phi(t)\right| \ll L_{M} \quad \text { as } \quad M \rightarrow \infty,
$$

where

$$
L_{M}:=\frac{D_{M}}{B_{M}^{1+\delta / 2}}
$$

and $\Phi$ is the standard normal distribution (For Lyapunov's central limit theorem, see for example $\$ 1.1$ and $\$ 1.2$ of [28]). Consequently we have

$$
\mathbb{P}\left(\left|\sum_{i=1}^{M} d_{i} Y_{i}\right| \geq \frac{\sqrt{B_{M}}}{\log M}\right) \rightarrow 1 \quad \text { as } M \rightarrow \infty,
$$

which together with (61) implies

$$
\underset{M \rightarrow \infty}{\limsup }\left|\sum_{i=1}^{M} d_{i} Y_{i}\right|=\infty \quad \text { a.e. }
$$

Now (57) and the first Borel-Cantelli lemma imply that we also have

$$
\underset{M \rightarrow \infty}{\limsup }\left|\sum_{i=1}^{M} \sum_{k \in \Gamma_{i}} c_{k} f_{\alpha}\left(n_{k} x\right)\right|=\infty \quad \text { a.e., }
$$

which implies

$$
\limsup _{N \rightarrow \infty}\left|\sum_{k=1}^{N} c_{k} f_{\alpha}\left(n_{k} x\right)\right|=\infty \quad \text { a.e. }
$$

This proves the optimality of Theorem 2 for almost everywhere convergence.

We note that a more detailed analysis shows that a possible choice for the constant $K_{1}$, and accordingly also for the constant $\hat{K}(\alpha)$ in the statement of Theorem 2 , is

$$
K_{1}=((2 \alpha-1) /(2 \alpha \log 2))^{1-\alpha}(1-\alpha)^{-1}-\varepsilon
$$

for an arbitrary $\varepsilon>0$. Consequently the "blowup" of the constant in the extra convergence condition is of order $(1-\alpha)^{-1}$ as $\alpha \rightarrow 1$, both in the sufficiency condition and in the optimality result.

Proof of Theorem 3: By (39) and Lemma 7 we have for any real sequence $\left(c_{k}\right)_{k \geq 1}$ and any $M, N$ satisfying $1 \leq M<N$ that

$$
\int_{0}^{1}\left(\sum_{k=M}^{N} c_{k} f(k x)\right)^{2} d x \ll \sum_{k, \ell=M}^{N}\left|c_{k} c_{\ell}\right| \frac{(\operatorname{gcd}(k, \ell))^{2 \alpha}}{(k \ell)^{\alpha}} \ll \sum_{k \leq N^{2}} \hat{b}_{k}^{2},
$$

where $\hat{b}_{k}$ are defined by

$$
\hat{b}_{k}=\frac{1}{k^{\alpha}} \sum_{d \mid k, d \geq M} d^{\alpha}\left|c_{d}\right|
$$


Let $\varepsilon>0$ be so small that $1-2 \alpha+\varepsilon<0$, and that (8) holds. For the simplicity of the formulas, in the sequel we write $\sigma(k)$ for $\sigma_{1-2 \alpha+\varepsilon}(k)$. By (63) and the Cauchy-Schwarz inequality we have

$$
\begin{aligned}
& \hat{b}_{k}^{2}=\left(\sum_{d \mid k, d \geq M}\left|c_{d}\right|(d / k)^{\alpha}\right)^{2} \\
& =\left(\sum_{d \mid k, d \geq M}\left|c_{d}\right|(d / k)^{\frac{1}{2}+\frac{\varepsilon}{2}}(k / d)^{-\alpha+\frac{1}{2}+\frac{\varepsilon}{2}}\right)^{2} \\
& \leq \sum_{d \mid k, d \geq M} c_{d}^{2}(d / k)^{1+\varepsilon} \sum_{d \mid k, d \geq M}(k / d)^{1-2 \alpha+\varepsilon} \\
& =\sum_{d \mid k, d \geq M} c_{d}^{2}(d / k)^{1+\varepsilon} \sum_{h \mid k, h \leq k / M} h^{1-2 \alpha+\varepsilon} \\
& \leq \sum_{d \mid k, d \geq M} c_{d}^{2}(d / k)^{1+\varepsilon} \sigma(k) .
\end{aligned}
$$

Thus

$$
\begin{aligned}
\sum_{k=M}^{N^{2}} \hat{b}_{k}^{2} & \leq \sum_{k=M}^{N^{2}} \sum_{d \mid k, d \geq M} c_{d}^{2}(d / k)^{1+\varepsilon} \sigma(k) \\
& \leq \sum_{d=M}^{N^{2}} c_{d}^{2} d^{1+\varepsilon} \sum \sigma(k) k^{-(1+\varepsilon)}
\end{aligned}
$$

where the inner sum is extended for all $k$ of the form $k=j d, j=1,2, \ldots$. But $\sigma(j d) \leq \sigma(d) \sigma(j)$ and thus the inner sum in (64) is bounded by

$$
\sum_{j=1}^{\infty} \sigma(d) \sigma(j)(d j)^{-(1+\varepsilon)} \ll \sigma(d) d^{-(1+\varepsilon)} \underbrace{\sum_{j=1}^{\infty} \sigma(j) j^{-(1+\varepsilon)}}_{\ll 1} \ll \sigma(d) d^{-(1+\varepsilon)},
$$

where we used the fact that $\sigma(j) \leq d(j)=O\left(j^{\eta}\right)$ for any $\eta>0$. Substituting this into (64), we get, together with (62), that

$$
\int_{0}^{1}\left(\sum_{k=M}^{N} c_{k} f(k x)\right)^{2} d x \ll \sum_{k=M}^{N^{2}} c_{k}^{2} \sigma(k)
$$

By (8) the right-hand side of (65) can be made arbitrarily small if $M$ is chosen sufficiently large. Thus by the Cauchy convergence test the series (3) is convergent in $L^{2}$.

To prove the second part of Theorem 3, let $\alpha \in(1 / 2,1), 0<\beta<1$, and choose $\delta>0$ so small that $\beta(1+\delta)<1$. Then by the second statement of Theorem 1 there exist a function $f \in C_{\alpha}$ and a sequence $\left(c_{k}\right)_{k \geq 1}$ such that

$$
\sum_{k=1}^{\infty} c_{k}^{2} \exp \left(\frac{\beta(1+\delta)}{1-\alpha} \frac{(\log k)^{1-\alpha}}{\log \log k}\right)<\infty
$$


but the series (3) does not converge in $L^{2}$ norm. In view of (11), the terms of the sum in (9) are smaller than those of (66) for sufficiently large $k$ and thus the sum (9) converges, proving the second half of Theorem 3.

\section{REFERENCES}

[1] C. Aistleitner. Convergence of $\sum c_{k} f(k x)$ and the Lip $\alpha$ class. Proc. Amer. Math. Soc., 140(11):3893-3903, 2012.

[2] C. Aistleitner, I. Berkes, and K. Seip. GCD sums from Poisson integrals and systems of dilated functions. J. Eur. Math. Soc., to appear. Available at http://arxiv . org/abs/1210.0741.

[3] I. Berkes. On the asymptotic behaviour of $S f\left(n_{k} x\right)$. Main theorems. Z. Wahrscheinlichkeitstheorie und Verw. Gebiete, 34(4):319-345, 1976.

[4] I. Berkes. On the convergence of $\sum c_{n} f(n x)$ and the Lip 1/2 class. Trans. Amer. Math. Soc., 349(10):41434158, 1997.

[5] I. Berkes and M. Weber. On series of dilated functions. Quart. J. Math., to appear. Available at http:// arxiv. org/pdf/1205.1326.

[6] I. Berkes and M. Weber. On the convergence of $\sum c_{k} f\left(n_{k} x\right)$. Mem. Amer. Math. Soc., 201(943):viii+72, 2009.

[7] I. Berkes and M. Weber. On series $\sum c_{k} f(k x)$ and Khinchin's conjecture. Israel J. Math., to appear.

[8] A. Bondarenko and K. Seip. GCD sums and complete sets of square-free integers. http: //arxiv . org/abs / 1402.0249 .

[9] J. Bourgain. Almost sure convergence and bounded entropy. Israel J. Math. 63 (1988), 79-95.

[10] J. Brémont. Davenport series and almost-sure convergence. Q. J. Math., 62(4):825-843, 2011.

[11] L. Carleson. On convergence and growth of partial sums of Fourier series. Acta Math., 116:135-157, 1966.

[12] H. Davenport. On some infinite series involving arithmetical functions. Q. J. Math., Oxf. Ser., 8:8-13, 1937.

[13] I. S. Gál. A theorem concerning Diophantine approximations. Nieuw Arch. Wiskunde (2), 23:13-38, 1949.

[14] V.F. Gapoškin Convergence and divergence systems. Mat. Zametki 4:253-260, 1968.

[15] T. H. Gronwall. Some asymptotic expressions in the theory of numbers. Trans. Amer. Math. Soc., 14:113-122, 1913.

[16] T. Hilberdink. An arithmetical mapping and applications to $\Omega$-results for the Riemann zeta function. Acta Arith., 139(4):341-367, 2009.

[17] S. Jaffard. On Davenport expansions. In Fractal geometry and applications: a jubilee of Benoît Mandelbrot. Part 1, volume 72 of Proc. Sympos. Pure Math., pages 273-303. Amer. Math. Soc., Providence, RI, 2004.

[18] S. Jaffard and S. Nicolay. Space-filling functions and Davenport series. In Recent developments in fractals and related fields, Appl. Numer. Harmon. Anal., pages 19-34. Birkhäuser Boston Inc., Boston, MA, 2010.

[19] A. J. Khinchin, A.J.: Ein Satz über Kettenbrüche mit arithmetischen Anwendungen. Math. Z. 18 (1923), 289306.

[20] M. Knopp and S. Robins. Easy proofs of Riemann's functional equation for $\zeta(s)$ and of Lipschitz summation. Proc. Amer. Math. Soc., 129(7):1915-1922, 2001.

[21] J. F. Koksma. On a certain integral in the theory of uniform distribution. Nederl. Akad. Wetensch., Proc. Ser. A. 54 = Indagationes Math., 13:285-287, 1951.

[22] J. F. Koksma. Estimations de fonctions à l'aide d'intégrales de Lebesgue, Bull. Soc. Math. Belg. 6 (1953), 4-13.

[23] L. B. Koralov and Y. G. Sinai. Theory of probability and random processes. Universitext. Springer, Berlin, second edition, 2007.

[24] P. Lindqvist and K. Seip. Note on some greatest common divisor matrices. Acta Arith., 84(2):149-154, 1998.

[25] J. M. Marstrand. On Khinchin's conjecture about strong uniform distribution. Proc. London Math. Soc. 21 (1970), 540-556.

[26] M. Mikolás. Integral formulae of arithmetical characteristics relating to the zeta-function of Hurwitz. Publ. Math. Debrecen, 5:44-53, 1957.

[27] H.L. Montgomery. Extreme values of the Riemann zeta function. Comment. Math. Helv., 52(4):511-518, 1977. 
[28] V. Petrov. Classical-type limit theorems for sums of independent random variables. In Limit theorems of probability theory. Transl. from the Russian by B. Seckler, pages 1-24. Berlin: Springer, 2000.

[29] K. Soundararajan. Extreme values of zeta and L-functions. Math. Ann., 342(2):467-486, 2008.

[30] M. J. G. Weber. On systems of dilated functions. C. R. Math. Acad. Sci. Paris, 349(23-24):1261-1263, 2011.

[31] A. Wintner. Diophantine approximations and Hilbert's space. Amer. J. Math., 66:564-578, 1944.

[32] A. Zygmund. Trigonometric series. Vol. I, II. Third edition. Cambridge University Press, Cambridge, 2002.

Department of Mathematics, Graduate School of Science, Kobe University, Kobe 657-8501, Japan

E-mail address: aistleitner@math. tugraz . at

Institute of Statistics, TU GraZ, KopernikUsgasse 24/III, 8010 GraZ, AUSTRIA

E-mail address: berkes@tugraz . at

Department of Mathematical Sciences, NorWEgian University of SCiEnCE and TeChnOlOgy (NTNU), NO-7491 TRONDHEIM, NORWAY

E-mail address: seip@math.ntnu.no

IRMA, 10 RUE du GÉnÉral Zimmer, 67084 Strasbourg CedeX, France

E-mail address: michel . weber@math . unistra.fr 